\title{
MATERNAL-FOETAL INCOMPATIBILITY ASSOCIATED WITH THE H-3 LOCUS IN THE MOUSE
}

\author{
PETER HULL \\ Department of Biology, University of Strathelyde, Glasgow
}

Received 15.v.68

\section{INTRODUCTION}

THE evolutionary consequences of maternal-foetal incompatibility, where offspring of the same genotype as their mother at some specific locus are at a selective disadvantage (autoincompatibility), have been considered. A stable genetic polymorphism involving two or more alleles at such a locus can exist, given certain restrictions on the selective parameters (Hull, $1964 a, b, 1966)$. It has been shown that immunological dissimilarity between mother and offspring favours placental development (Billington, 1964) and that the degree of enhancement of growth could depend on the extent of maternal sensitisation to a foetal antigen (James, 1965). It was suggested (Clarke and Kirby, 1966) that the observed deficiencies of offspring of coat colour like that of their mother (Hull, 1964b) could be explained in terms of the effect, not of the genes at the $a$ (agouti) locus controlling coat colour, but of closely linked histocompatibility genes. The $H-3$ locus is within 10 cross-over units of the $a$ locus (Snell, 1958), as is at least one other weaker histocompatibility locus (Snell and Bunker, 1964). It appeared reasonable to test whether or not there was an autoincompatibility effect associated with segregation at the $H-3$ locus in an otherwise genetically uniform strain of mice.

\section{EXPERIMENTAL MATERIAL}

Mice of the strain Bl0.LP, homozygous for a chromosome segment bearing the genes $A^{w}$ (at the $a$ locus) and $H-3 b$ (at the $H-3$ locus), introduced from strain LP on to a C57BL10/ScSn genetic background (Snell and Bunker, 1964) were crossed with $\mathrm{C} 57 \mathrm{BL} 10 / \mathrm{ScSn}$ mice (homozygous a, $\mathrm{H}-3 a$ ), hereafter called C57. The experimental crosses, the results of which are described later, were obtained by backcrossing the $F_{1}$ mice either to strain C57 or to strain B10.LP mice. In the first case $H-3 a / H-3 a$ and $H-3 a / H-3 b$ progeny would be expected in equal numbers. In fact, about 90 per cent. of the $H-3 a / H-3 a$ progeny will be $a a$ (black) and 90 per cent. of the $H-3 a / H-3 b$ progeny will be $A^{w} a$ (white-bellied agouti). It was decided not to attempt to tissue-type offspring from this cross but to use their coat colour phenotype as an imperfect indicator of their $H-3$ type. In the case of the cross of $F_{1}$ mice with B10.LP, all progeny would be expected to be whitebellied agouti ( $\frac{1}{2} A^{w} a$ and $\frac{1}{2} A^{w} A^{w}$ ). To determine whether these mice were $\mathrm{H}-3 \mathrm{a} / \mathrm{H}-3 b$ or $\mathrm{H}-3 b / \mathrm{H}-3 b$ all were pre-immunised with three injections of normal C57 thymus cells (from 4-week-old donors) at weekly intervals (from $4 \cdot 2 \times 10^{6}$ to $6 \cdot 6 \times 10^{6}$ cells in $0 \cdot 1 \mathrm{ml}$. tissue culture medium at the first injection and around $3 \times 10^{6}$ cells at the other two) and challenged one week later with a subcutaneous injection of an ascitic leukemia derived from C57, known as E.L.4 (Gorer, 1950), as $0.4 \times 10^{6}$ to $0.42 \times 10^{6}$ cells in $0.1 \mathrm{ml}$. 
tissue culture medium. It would be expected that $H-3 a / H-3 b$ mice would accept this tumour, and die, whereas $H-3 b \mid H-3 b$ mice would reject the tumour (Snell and Bunker, 1964). The mice were from 8 to 12 weeks old at the time of the first thymus injection. The testing was done in three lots and one cage of 6-8 C57 mice and one containing 6-8 B10.LP mice were

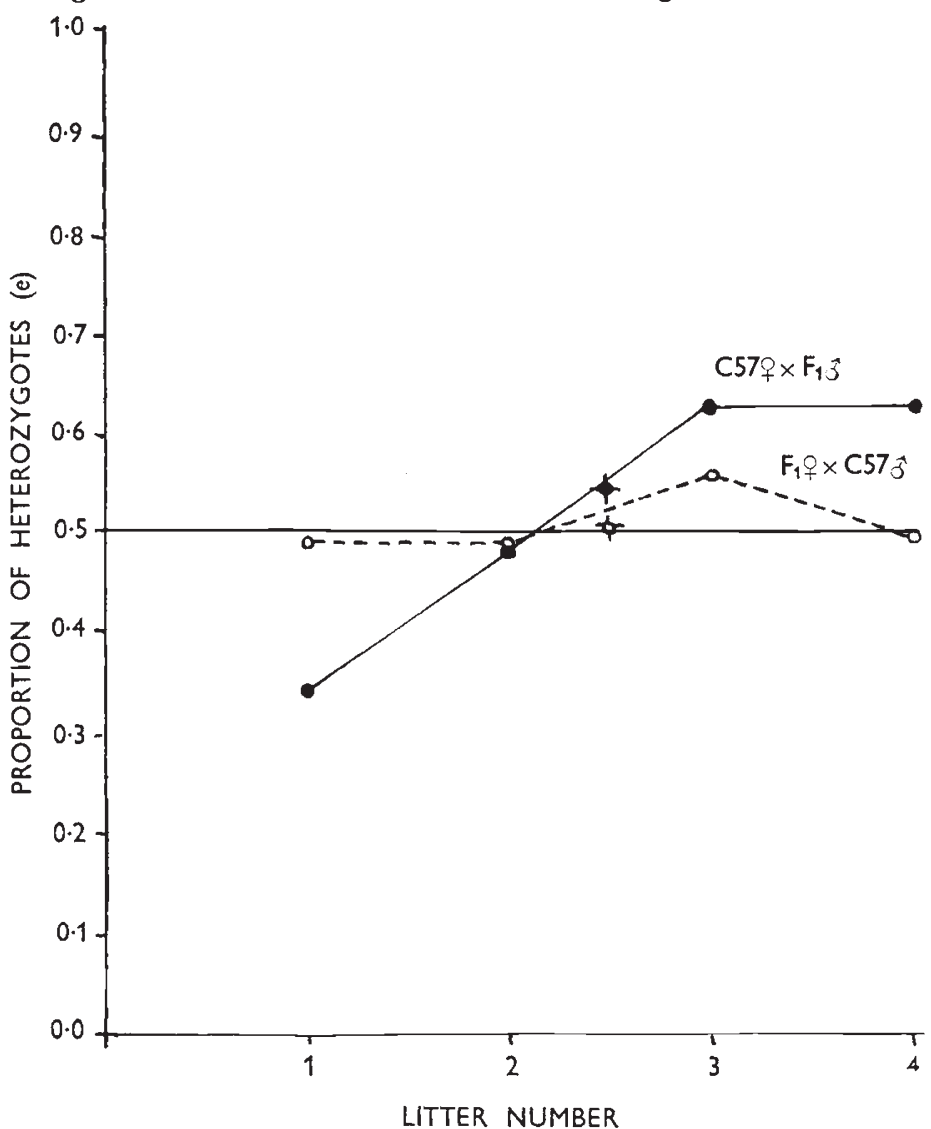

FIG. 1.-Proportion of heterozygous offspring from the mating $a a q \times a A^{w} \sigma^{*}$ (solid circles) and its reciprocal (open circles). That is

$$
e=\frac{\text { no. } a A^{w}}{\text { no. } a A^{w}+\text { no. } a a}
$$

treated and challenged at the same time. All C57 mice died and all B10.LP mice survived. In addition, at the end of the tests, as part of another experiment, 30 known $H-3 b / H-3 b$ mice were given three injections of C57 thymus and challenged with E.L.4 in a similar way. All survived. There was thus no evidence to suggest any change in antigenic properties of the tumour during the period of the experiment, when it was being transferred in G57 mice (14 transfers in all).

Twelve matings of each of the following types were made:

$$
\begin{aligned}
& \mathrm{C} 57 \text { 우 } \times \mathrm{F}_{1} \text {, } \\
& \mathrm{F}_{1} \text { 우 } \times \mathrm{C} 57 \text { 중, } \\
& \mathrm{F}_{1} \text { 우 } \times \text { B10.LPA } \text { A }^{\text {, }} \\
& \text { Bl0.LP우 } \times \mathrm{F}_{1} \mathrm{O}^{x} \text {. }
\end{aligned}
$$


Half of the $F_{1}$ mice used as parents in each of the four types of cross were derived from the mating $\mathrm{C} 57 \% \times \mathrm{B} 10 . \mathrm{LP}_{\bigcirc}$ and half from the reciprocal cross $\mathrm{B} 10 . \mathrm{LP}$ + $\times \mathrm{C} 57$ \%

The matings were all made on the same day and discarded when they had produced four litters, or after 21 weeks. All offspring were weighed at

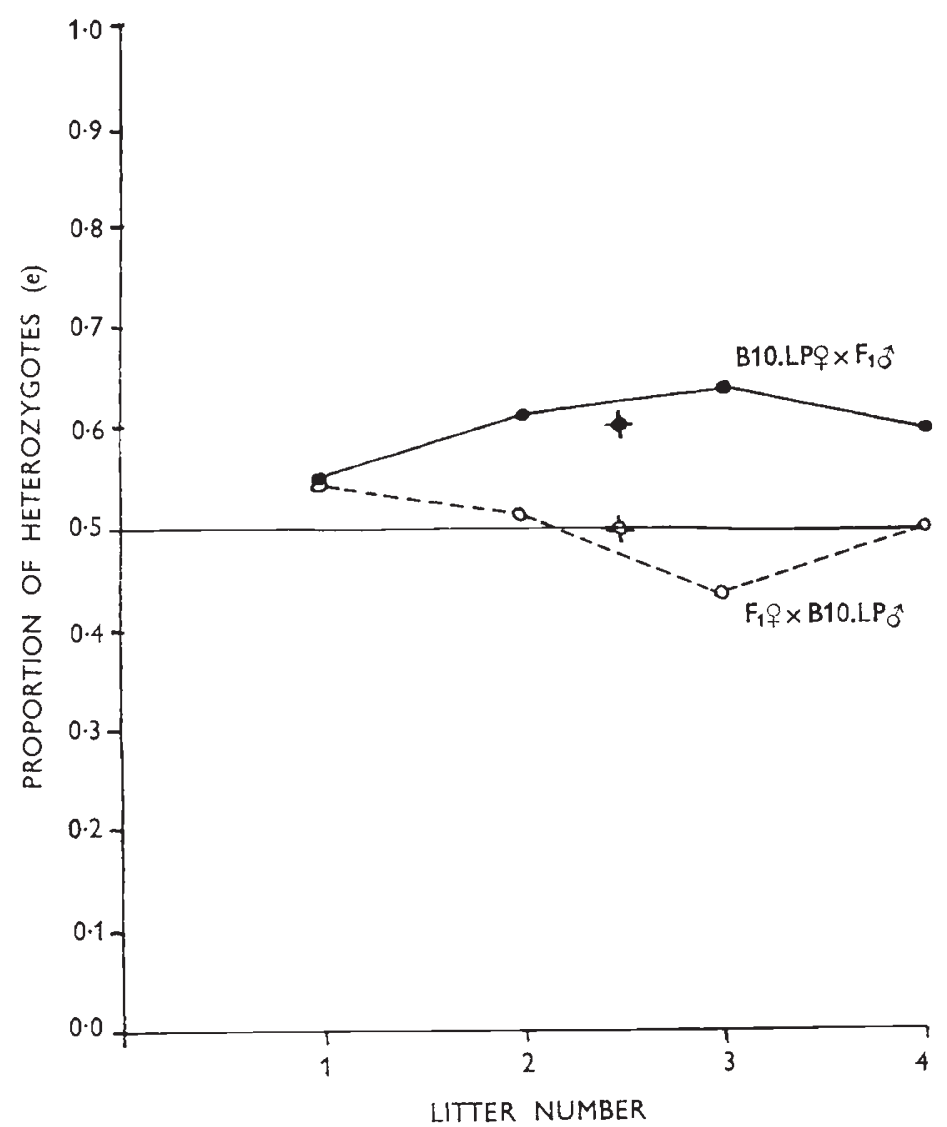

FIG. 2.-Proportion $(e)$ of offspring which are heterozygous from the mating of $H-3 a / H-3 b q \times H-3 b / H-3 b{ }^{\star}$

(open circles) and its reciprocal (solid circles). That is

$$
e=\frac{\text { no. } H-3 a / H-3 b}{\text { no. } H-3 a / H-3 b+\text { no. } H-3 b / H-3 b}
$$

birth and toe clipped so that they could be identified later. Offspring were weaned at 3 weeks of age, when they were reweighed. Data were included only for those animals which survived to weaning and were classified.

\section{Results}

In each type of mating the expected ratio of heterozygous to homozygous offspring ( $a a$ or $H-3 b / H-3 b$ ) is $1: 1$. In figs. 1 and 2 are plotted the proportion $(e)$ of the total number of offspring $(n)$ which were heterozygous in first, second, third and fourth litters. 
In table 1 these results are shown, together with $\chi^{2}$ tests of the hypotheses that the observed ratios are not significantly different from 0.5 .

In order to test the hypotheses that there were no differences in weight at birth or weaning between heterozygous and homozygous offspring from the four types of mating, $t$-tests were performed. Since there were large differences in mean weight between litters (partly a reflection of the different numbers of offspring in the litters or the parity of the female), and between the two sexes, weights of male and female offspring were treated separately and the analysis was confined to those $(m)$ litters in a particular cross in which both homozygous and heterozygous offspring of the sex under

TABLE 1

Proportion (e) of heterozygotes, together with $\chi^{2}$ with one degree of freedom

$$
\begin{aligned}
& \chi_{(1)}^{2} 5 \%=3.84 \\
& \chi_{(1)}^{2} 1 \%=6.63
\end{aligned}
$$

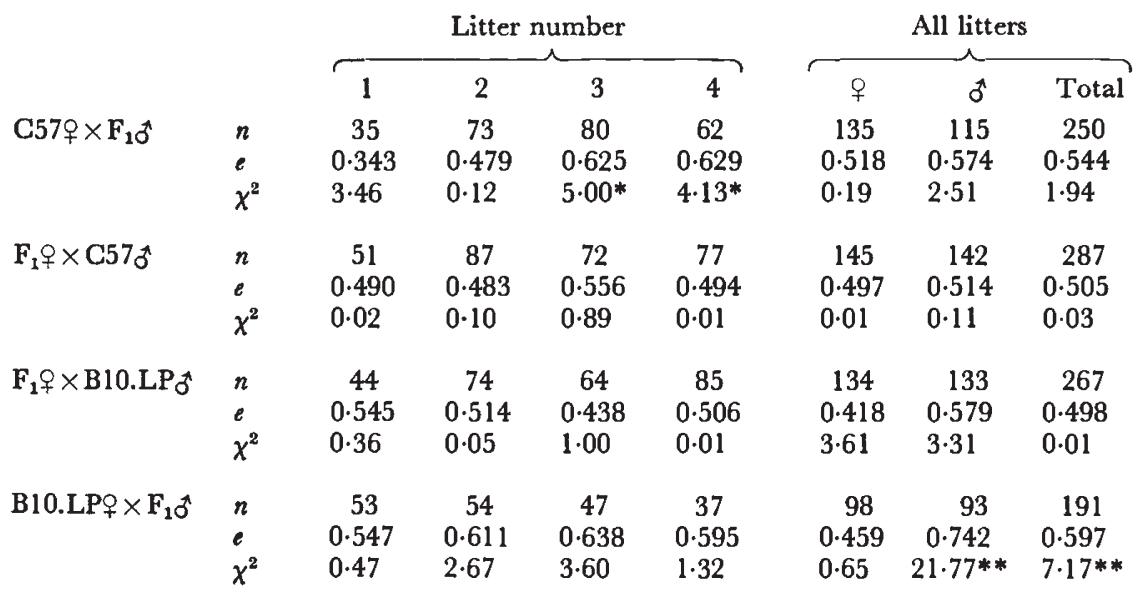

consideration were produced. The $(m)$ within-litter differences between weights at a particular age of homozygotes and heterozygotes were obtained: for example, difference 1 = mean weight of homozygous offspring in litter 1 minus mean weight of heterozygous offspring in litter 1 . The mean of these differences was then calculated for that particular cross and divided by its standard error to give an estimate of $t$ with $m-1$ degrees of freedom.

\section{INTERPRETATION OF THE DATA}

\section{(i) Genotypic ratios among offspring of different crosses}

It may be seen from fig. 1 that there is an excess of heterozygous offspring (unlike their mother in genotype) from the crosses of $a a+\times a A^{w} \hat{o}^{*}$ and $A^{w} A^{w}$ o $\times a A^{w}{ }^{*}$. This excess is highly significant when the total of all offspring is considered in the cross of $A^{w} A^{w} q \times a A^{w}$, but is only significant in litters three and four from the cross of $a a$ 우 $\times a A^{w} \hat{\delta}$. Indeed, in the first litters from this cross the probability of obtaining by chance a deficiency of heterozygotes as great as, or greater than, that observed $(e=0.343)$ is only about 0.07 . 
There is significant difference in $e$ ratio amongst the four litters in this cross $\left(x_{(3)}^{2}=10 \cdot 86, \mathrm{P}<0 \cdot 05\right)$. There is, however, no significant difference in $e$ ratio (based on the data from the total of all four litters) between the sexes among the offspring $\left(\chi_{(1)}^{2}=0 \cdot 77\right)$ nor between the sexes for the total data from the third and fourth litters only $\left(\chi_{(1)}^{2}=0.34\right)$. It appears that there may be preferential survival of $a A^{w}(H-3 a / H-3 b)$ offspring rather than $a a(H-3 a / H-3 a)$ in the later litters from aa $(H-3 a / H-3 a)$ mothers. This autoincompatibility effect is not shown among $a a(H-3 a / H-3 a)$ and $a A^{w}(H-3 a / H-3 b)$ offspring of $a A^{w}(H-3 a / H-3 b)$ mothers from the second cross.

There is a clear indication of preferential survival of heterozygous $(H-3 a / H-3 b)$ offspring rather than homozygous $(H-3 b / H-3 b)$ offspring from homozygous $(H-3 b / H-3 b)$ mothers in the fourth cross (total $e=0.597$. $\left.x_{(1)}^{2}=7 \cdot 17,0.005<\mathrm{P}<0.01\right)$. There is no similar discrimination among offspring from $H-3 a / H-3 b$ females (third cross). But this superiority appears only among male offspring $\left(e\left(\delta^{\prime}\right)=0.742, \chi_{(1)}^{2}=21.37, \mathrm{P}<0.001\right)$. Among the female offspring $e$ is less than $0 \cdot 500$, though not significantly so: there is a highly significant difference in $e$ ratio between the sexes in this cross $\left(x_{(1)}^{2}=12.55, \mathrm{P}<0.001\right)$. In fact $e\left(\delta^{\prime}\right)$ is greater than 0.500 for all crosses, though significantly so only in the last one. It appeared that the most conservative estimate of the significance of the increase of $e$ among males from the last cross could be obtained by comparing this ratio with the next highest, that is, with that among male offspring from the third cross, where $e(\delta)$ is greater than 0.500 , but not significantly so. There is a significant difference in $e$ ratio between males from these two crosses $\left(\chi_{(1)}^{2}=6 \cdot 35\right.$, $\mathrm{P}<0 \cdot 01)$.

\section{(ii) Differences of weight at birth and weaning from different crosses}

None of the differences, within litter in weight at birth or weaning between homozygous and heterozygous animals of either sex in any of the four crosses was significant.

\section{Discussion}

The autoincompatibility observed between homozygous mothers and offspring is similar to that previously seen when the $a^{t} /+$ system was studied. There is however no evidence of any autoincompatibility between heterozygous mothers and their offspring. The inability of a heterozygous mother to distinguish between the antigens of heterozygous and homozygous offspring would not be unexpected.

The autoincompatibility effect is significant only in later litters in the first cross. There could be a resemblance between this effect, if real, and other reactions between mother and foetus (such as that producing $\mathrm{Rh}$ haemolytic disease), which increase in intensity with increasing parity.

It is generally considered that the sex-associated graft rejection within strain C.57 (Eichwald et al., 1958) is due to a $\Upsilon$-linked gene controlling the production of a histocompatibility antigen. If histocompatibility differences are involved in producing autoincompatibility, it is conceivable that there could be a multiplicative interaction between factors in determining the degree of autoincompatibility in some circumstances. The result of 
cross four (table 1), where autoincompatibility is apparently confined to male offspring, could be regarded as some evidence for a hypothesis of nonadditive reinforcement.

Half of the $F_{1}$ animals in each cross were derived from the mating of C57우 with B10.LP ${ }^{\star}$ and half from the reciprocal mating. Half of the males in the first and last crosses thus had a $Y$ chromosome derived from Bl0.LP and the other half, one from C57. There was no evidence of heterogeneity of $e$ ratio among female offspring $\left(\chi_{(1)}^{2}=2 \cdot 34\right)$ or male offspring $\left(\chi_{(1)}^{2}=1.79\right)$ from males of the two types in the first cross or among female offspring $\left(\chi_{(1)}^{2}=0 \cdot 12\right)$ or male offspring $\left(\chi_{(1)}^{2}=0 \cdot 34\right)$ from males of the two types in the last cross. There is no evidence that the $r$ chromosome is not the same in its effects in strains C57 and B10.LP and it is reasonable to pool the data, as in table 1 .

None of the differences, within litter, in weight at birth or weaning in either sex is significant, even in the case of offspring from the first and last cross, where there is evidence of autoincompatibility. It is possible that differential mortality, if associated with autoincompatibility, takes place early after conception, and that after this stage growth proceeds normally among survivors. It is also possible that real differences exist but that the number of mice weighed in this experiment was not great enough to detect them.

\section{SUMmary}

1. Crosses of $H-3 a / H-3 a$ or $H-3 b / H-3 b$ females with congenic $H-3 a / H-3 b$ males produced significantly less than 50 per cent. of homozygous offspring.

2. This deficiency was found only among offspring from later litters from $H-3 a / H-3 a$ females and only among male offspring from $\mathrm{H}-3 b / \mathrm{H}-3 b$ females.

3. No similar disturbance of segregation among offspring of heterozygous females was noted.

4. No significant differences in weight at birth or weaning were found between homozygous and heterozygous offspring in any of the crosses.

Acknowledgments.-Dr J. R. Batchelor kindly provided a sample of E.L.4. Part of this work was done during the tenure of a research fellowship at the University of Liverpool, where Professor P. M. Sheppard gave much valued help and advice.

\section{REFERENCES}

BILLINGton, w. D. 1964. Influence of immunological dissimilarity of mother and foetus on the size of placenta in mice. Nature, 202, 317-318.

CLARKE, B., AND KIRBY, D. R. s. 1966. Maintenance of histocompatibility polymorphisms. Nature, 211, 999-1000.

EICHWALD, E. J., SILMSER, C. R., AND WEISSMAN, I. 1958. Sex linked rejection of normal and neoplastic tissue. F. Nat. Cancer Inst., 20, 563-575.

GORER, P. A. 1950. Studies in antibody response of mice to tumour inoculation. Brit. $\mathbf{F}$. Cancer, 4, 372-379.

HULL, P. 1964a. Equilibrium of gene frequency produced by partial incompatibility of offspring with dam. P.N.A.S., 51, 461-464.

HUll, P. 1964b. Partial incompatibility not affecting total litter size in the mouse. Genetics, 50, 563-570.

HULL, P. 1966. Possible stability of polymorphism in a multiallele incompatibility system in the mouse. Genetics, 54, 1049-1053. 
JAMES, D. A. 1965. Effects of antigenic dissimilarity between mother and foetus on placental size in mice. Nature, 205, 613-614.

SNELL, G. D. 1958. Histocompatibility genes of the mouse. I. demonstration of weak histocompatibility differences by immunisation and controlled tumour dosage. $\mathcal{Z}$. Nat. Cancer Inst., 20, 787-824.

SNELL, G. D., AND BUNKER, H. 1964. Histocompatibility genes of the mouse. IV. The position of $H-3$ in the fifth linkage group. Transplantation, 2, 743-751. 